\title{
Impact of Shape of Obstacle Roof on the Turbulent Flow in a Wind Tunnel
}

\author{
Slah Driss, Zied Driss ${ }^{*}$, Imen Kallel Kammoun \\ Laboratory of Electro-Mechanic Systems (LASEM), National School of Engineers of Sfax (ENIS), Univrsity of Sfax, TUNISIA \\ *Corresponding author: zied.driss@enis.rnu.tn
}

Received September 13, 2014; Revised October 08, 2014; Accepted October 09, 2014

\begin{abstract}
In this paper, we are interested in the impact of shape of obstacle roof on the turbulent flow in a wind tunnel. Particularly,arched, inclined, pitched and flat roofs obstacles are examined. A three-dimensional flow of a fluid is numerically analyzed using the Navier-Stokes equations in conjunction with the standard k- $\varepsilon$ turbulence model. These equations were solved by a finite-volume discretization method. The comparison between our numerical and experimental results shows a good agreement and confirms the numerical method.
\end{abstract}

Keywords: modeling, turbulent flow, roof shape, obstacle, wind tunnel

Cite This Article: Slah Driss, Zied Driss, and Imen Kallel Kammoun, "Impact of Shape of Obstacle Roof on the Turbulent Flow in a Wind Tunnel.” American Journal of Energy Research, vol. 2, no. 4 (2014): 90-98. doi: 10.12691/ajer-2-4-3.

\section{Introduction}

Understanding dispersion processes in urban areas is important for modelling air quality as well as pollution from accidental or terrorist releases. The chaotic nature of turbulent flow and the complexity of the building geometry both contribute to making such modelling nontrivial. Urban geometry affects the mean flow and turbulence significantly [1] and there by exerts a strong control on dispersion processes [2]. In this context, Ntinas et al. [3] predicted the airflow around buildings that is challenging due to the dynamic characteristics of wind. A time-dependent simulation model has been applied for the prediction of the turbulent airflow around obstacles with arched and pitched roof geometry, under wind tunnel conditions. To verify the reliability of the model an experiment was conducted inside a wind tunnel and the air velocity and turbulent kinetic energy profiles were measured around two small-scale obstacles with an arched-type and a pitched-type roof. Luo et al. [4] studied models of cuboid obstacles to characterize the three-dimensional responses of airflow behind obstacles with different shape ratios to variations in the incident flow in a wind-tunnel simulation. Wind velocity was measured using particle image velocimetry (PIV). The flow patterns behind cuboid obstacles were complicated by changes in the incidence angle of the approaching flow and in the obstacle's shape ratio. Ahmad et al. [5] provided a comprehensive literature on wind tunnel simulation studies in urban street canyons/intersections including the effects of building configurations, canyon geometries, traffic induced turbulence and variable approaching wind directions on flow fields and exhaust dispersion. Jiang et al. [6] studied three ventilation cases, single-sided ventilation with an opening in windward wall, single-sided ventilation with an opening in leeward wall, and cross ventilation. In the wind tunnel, a laser Doppler anemometry was used to provide accurate and detailed velocity data. In LES calculations, two subgrid-scale (SS) models, a Smagorinsky SS model and a filtered dynamic SS model, were used. The numerical results from large eddy simulation (LES) are in good agreement with the experimental data, in particular with the predicted airflow patterns and velocities around and within, and the surface pressures over the models. Nozawa et al. [7] studied a flow around an obstacle under the condition that a turbulent flow is approaching the obstacle. The turbulent inflow data were generated for both a smooth surface and a rough surface by the proposed method. A half-height cube was immersed in the turbulent boundary layers with several types of vertical velocity profile. Becker et al. [8] studied the structure of the flow field around three-dimensional obstacles of different aspect ratios in two different types of boundary layers. The dimensions of the rectangular block obstacles were chosen to represent generic shapes of buildings. In order to study the flow field around a building structure in a wind tunnel test, the simulation of a boundary layer similar to the atmospheric boundary layer was a crucial issue. Ikhwan et al. [9] conducted an experimental investigation of the flow and pressure chracterestics around pyramidal buildings in a boundary layer wind tunnel. The velocities of the flow around the pyramids and the pressure distribution on the pyramid surfaces were measured using 2D laser Doppler anemometry (LDA) and standard pressure tapping technique, respectively. Eight different pyramids (model scale, 1:200) with varying base angles were investigated. The mean and fluctuating characteristics that distinguish pyramids field around pyramids surfaces are described. Kenjeres et al. [10] observed for flows over submerged solid obstacles. It is demonstrated that 
complex flow patterns can be generated by imposing magnetic fields of different strengths. The initial validation of the electromagnetically extended Navier-Stokes solver on unstructured numerical grids is performed in the lowReynolds number range $100<\operatorname{Re}<400$ for different values of the magnetic interaction parameter. Santos et al. [11] used a mathematical model based on the solution of equations of conservation of mass, linear momentum and energy with the use of a non-standard $k-\varepsilon$ turbulence model. The modifications propsed in the k-cmodel are the inclusion of the Kato and Launder correction in the production of turbulent kinetic energy and the use of a modified wall function. Franke et al.[12] developed a computational study to solve the Reynolds Averaged Navier-Stokes equations with the standard k- $\varepsilon$ turbulence model. Five cases are computed comprising a 2D beam, a wall mounted cube rotated by $45^{\circ}$ against the approach flow, a cuboid and an array of 21 cuboids. A block structured hexahedral, an unstructured tetrahedral and an unstructured hybrid mesh of tetrahedral and prisms were used for each case. The computed mean velocity components were compared with corresponding experimental results. Sadaand Sato [13] developed a numerical simulation model to predict the instantaneous concentration fluctuation of a plume and applied to stack-gas diffusion around a cubical building. The flow field, including an instantaneous velocity component, was predicted using the large eddy simulation (LES) method in the developed numerical model. Tominaga and Stathopoulos [14] reviewed current modeling techniques in CFD simulation of near-field pollutant dispersion in urban environments and discussed the findings to give insight into future applications. De Paepe et al. [15] simulated five different wind incidence angles using a turntable in order to quantify their effect on indoor air velocities. The responses in local air velocities could largely be attributed to the relative position of the end walls of the scale models orientated towards the wind. This crucial position allows the measured air velocity trends to be explained. The estimated airflow rates gradually decreased for larger wind incidence angles. Lim et al. [16] presented a numerical simulation of flow around a surface mounted cube placed in a turbulent boundary layer which, although representing a typical wind environment. The simulations were carried out at a Reynolds number, based on the velocity at the cube height, of 20,000. The results presented include detailed comparison between measurements and LES computations of both the inflow boundary layer and the flow field around the cube including mean and fluctuating surface pressures. Melo et al. [17] developed two Gaussian atmospheric dispersion models, AERMOD and CALPUFF. Both incorporating the PRIME algorithm for plume rise and building downwash, are inter compared and validated using wind tunnel data. The results show that concentrations predicted by AERMOD are in general higher than those predicted by CALPUFF, especially regarding the maximum mean concentrations observed in the near field. Comparison of the model results with wind tunnel data showed that both models adequately predict mean concentrations further downwind from the facility. However, closer to the buildings, the models may overpredict or under-predict concentrations by a factor of two, and in certain cases even larger depending on the conditions.
According to these studies, it's clear that the study of the aerodynamic around the obstacle is very interesting. Indeed, the literature review confirms that there is paucity on the shape roof obstacle studies. Thus, we are interested in this work on the impact of shape of obstacle roof on the turbulent flow in a wind tunnel.

\section{Cases Study}

Figure 1 shows the test vein equipped by the considered obstacles with arched, inclined, pitched and flat roofs. The geometrical arrangements of these obstacles are presented is Figure 2. The characteristics parameters are defined by the lengths $\mathrm{l}=118 \mathrm{~mm}$ and the height $\mathrm{H}=153 \mathrm{~mm}$. To characterize the Inclined roof, we have introduced the second height $\mathrm{h}=82 \mathrm{~mm}$, for the arched and pitched roofs, we have introduced the second height clearance $c=20 \mathrm{~mm}$.

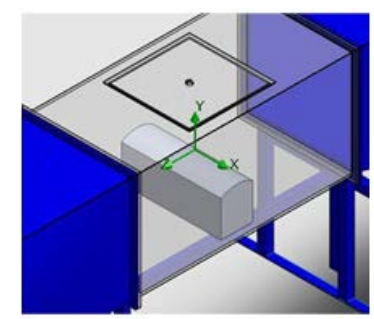

(a) Arched roof

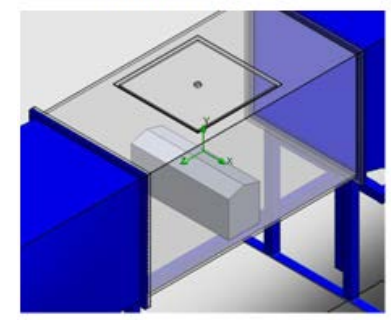

(c) Pitched roof

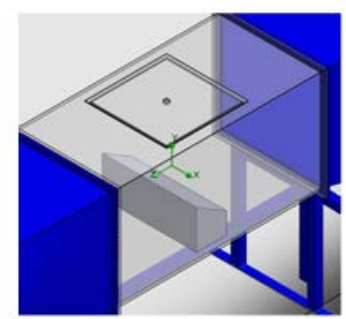

(b) Inclined roof

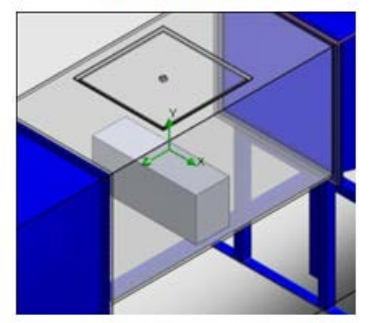

(d) Flat roof
Figure 1. Computational domain

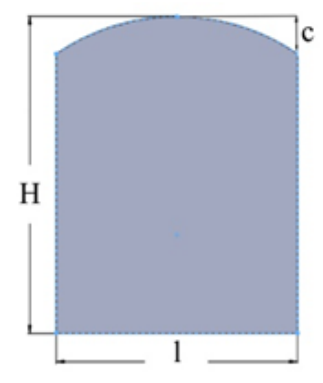

(a) Arched roof

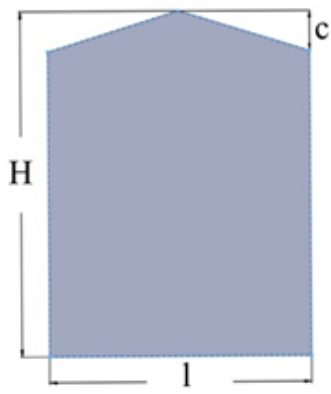

(c) Pitched roof

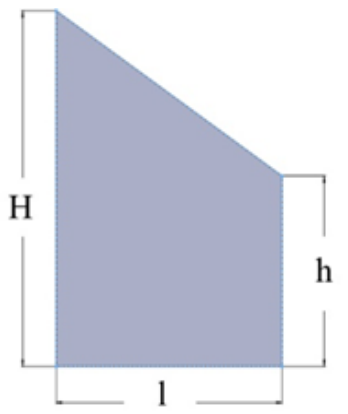

(b) Inclined roof

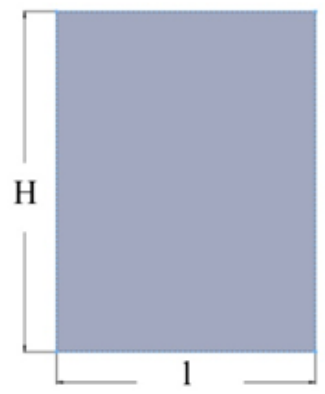

(d) Flat roof
Figure 2. Geometrical arrangements 


\section{Numerical Model}

The used computational fluid dynamic (CFD) code, "Solid works Flow simulation" combines a high level of functionality and accuracy with ease-of-use. This code is based on solving Navier-Stokes equations with a finite volume discretization method. The technique consists in dividing the computational domain into elementary volumes around each node in the grid; it ensures continuity of flow between nodes. The spatial discretization is obtained by following a procedure for tetrahedralinterpolation scheme. As for the temporal discretization, the implicit formulation is adopted. The transport equation is integrated over the control volume [18-23].

\subsection{Boundary Conditions}

The boundary conditions are required anywhere fluid enters the system and can be set as a pressure, mass flow, volume flow or velocity. For the inlet velocity we will take as a value $0.9 \mathrm{~m} . \mathrm{s}^{-1}$ and for the outlet pressure a value of $101325 \mathrm{~Pa}$. The same conditions are applied for the different considered case studies as shown in Figure 3. Knowing that the obstacle is suspended in our domain, both the roof top obstacle and the wall of our domain are considered as a wall boundary condition.

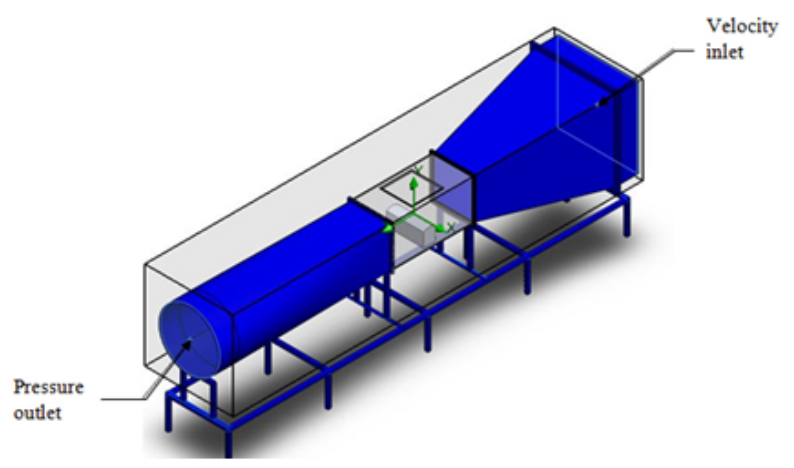

\begin{tabular}{|c|c|c|}
\hline Roof shape & Velocityinlet $\left(\mathrm{m} . \mathrm{s}^{-1}\right)$ & Pressure outlet $(\mathrm{Pa})$ \\
\cline { 1 - 1 } Inclined roof & & \\
\cline { 1 - 1 } Flat roof & 0.9 & 101325 \\
\cline { 1 - 1 } Pitched roof & & \\
\cline { 1 - 1 } Arched roof & & \\
\hline
\end{tabular}

Figure 3. Boundary conditions

\subsection{Meshing}

Figure 4 shows the mesh in the longitudinal plane defined by $x=0$. The mesh is constructed from the basic mesh by refining the basic mesh cells in accordance with the specified mesh settings. The basic mesh is formed by dividing the computational domain into slices by parallel planes which are orthogonal to the global coordinate system's axes. Grid-independency of the computational results is discussed in anterior papers. For example, we can refer to the paper [18]. In fact, the size of the mesh has been changed and the obtained results have been compared to the experimental velocity values collected from the test section of the wind tunnel. In particular, six meshes have been studied. The first case to be treated corresponds to a cell of $40 \mathrm{~cm}$. The second case corresponds to a cell of $10 \mathrm{~cm}$. The third case corresponds to a cell of $5 \mathrm{~cm}$. The fourth case corresponds to a cell of 2 $\mathrm{cm}$. The fifth case corresponds to a cell of $0.5 \mathrm{~cm}$. The latter one corresponds to a cell of $0.1 \mathrm{~cm}$. In all these cases, the number of cells is respectively equal to 1348, 3358, 5635, 10310, 37075 and 193701; which corresponds to a coarse mesh in the first case and a refined mesh in the sixth case. According to the obtained results, it has been noted that the velocity value obtained for the fifth case is the closest to the experimentally measured value. Also, it has been noted that the resolution time increases with the decrease of the size of mesh cells. The different velocity profiles seem to have the same appearance but the velocity values depends on the cell size. Indeed, the greater the cell size gets the more the gap between numerical and experimental results is large. The best result regarding precision and time is found to be a cell of $5 \mathrm{~mm}$ size. This choice leads to a better result with regards to the precision and the resolution time.

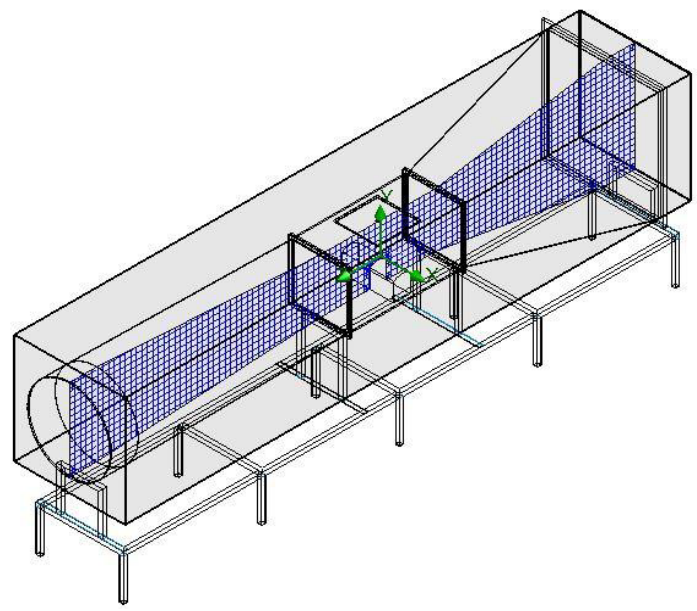

Figure 4. Meshing in the longitudinal plane $x=0$

\section{Numerical Results}

\subsection{Velocity Magnitude}

Figure 5 presents the distribution of the velocity magnitude in the longitudinal plane defined by $\mathrm{X}=0 \mathrm{~mm}$. According to these results, it has been noted that the velocity is weak in the inlet of the collector. It is indeed governed by the boundary conditions value of the inlet velocity which is equal to $0.9 \mathrm{~m} . \mathrm{s}^{-1}$. In this region, the velocity field is found to be uniform and increases progressively downstream of the collector. At the test vein, an important increase has been noted due to the reduction of the tunnel section that causes the throttling of the flow. While the upper side of the obstacle is characterized by the high velocity, a brutal drop is located behind the obstacle. This fact is due to the deceleration of the velocity field near the obstacle. In the test vein, the velocity keeps increasing till the out of the test section. Then, a decrease has been noted through the diffuser where the minimum velocity values are recorded in the lateral walls of the diffuser. Also, a wake characteristic of the maximum value appears in the gap localized between the test vein surface and the obstacle. In these conditions, 
it has been noted that the maximum value of the velocity is found with the flat roof model. This fact is due to the
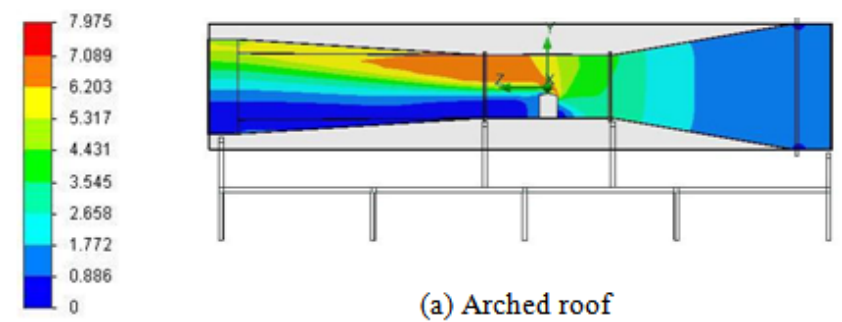

(a) Arched roof
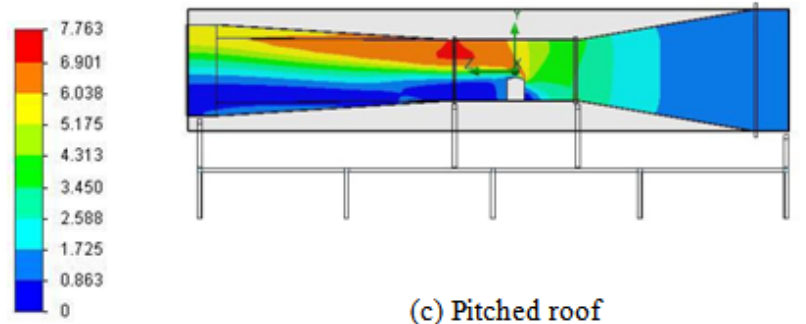

(c) Pitched roof geometry characteristics causing the perturbation of the air flow.

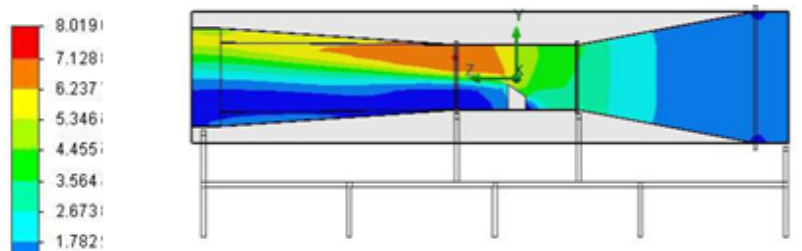

(b) Inclined roof

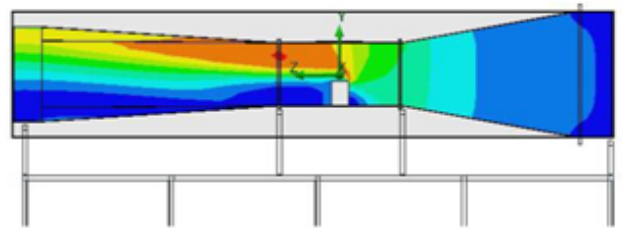

(d) Flat roof

Figure 5. Distribution of the magnitude velocity

\subsection{Velocity Streamlines}

Figure 6 presents the distribution of the velocity streamlines in the longitudinal plane defined by $\mathrm{X}=0 \mathrm{~mm}$. According to these results, it has been noted that the velocity is weak in the inlet of the collector. Indeed, it is governed by the boundary condition value of the inlet velocity. In this region, the velocity field is found to be uniform and increases progressively downstream of the collector. At the test vein, an important increase has been noted due to the reduction of the tunnel section that causes the throttling of the flow. While the upper side of the obstacle is characterized by the high velocity, a brutal drop is located behind the obstacle. This fact is due to the deceleration of the velocity field while passing by the obstacle. In the test vein, the velocity keeps increasing till the out of the test section. Then, a decrease on the maximum values has been noted through the diffuser where the minimum velocity values are recorded in the lateral walls of the diffuser. Indeed, a recirculation zone is located behind the obstacle. Besides, it has been noted that the dead zone is more developed in the region behind the inclined and the flat roof obstacles than the arched and the pitched roof obstacles. Also, it's clear that the wake characteristic of the maximum values appears in the gap localized between the test vein surface and the obstacle.
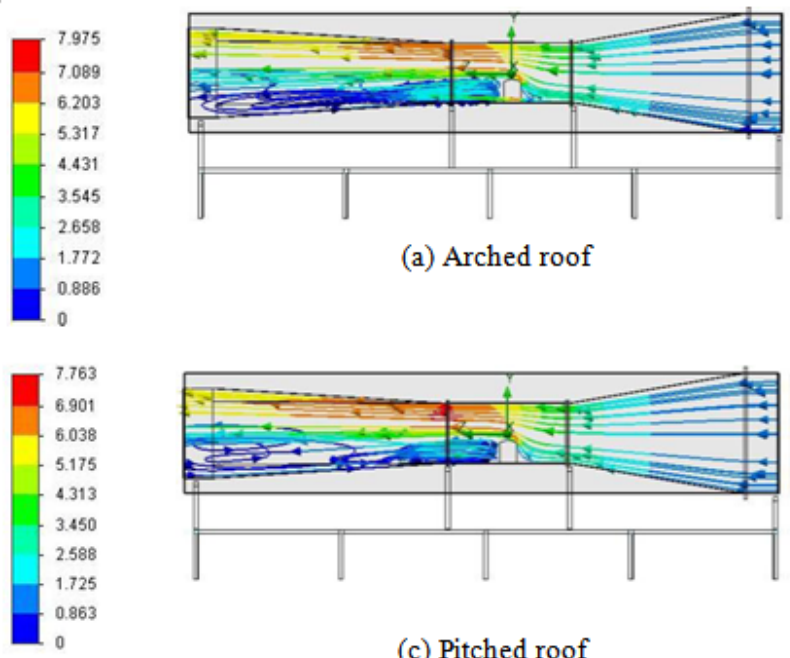

(a) Arched roof

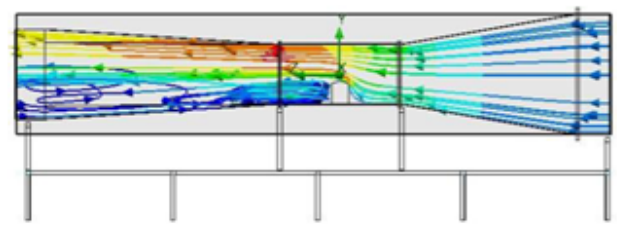

(c) Pitched roof
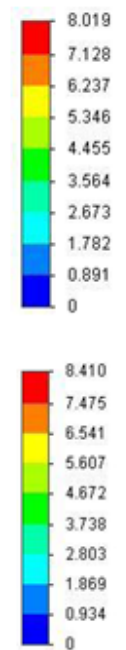

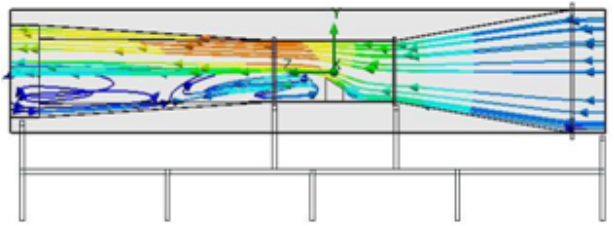

(b) Inclined roof

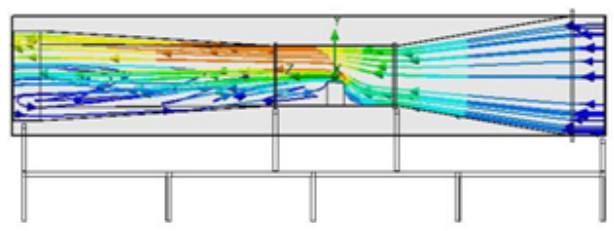

(d) Flat roof

Figure 6. Distribution of the velocity streamlines

\subsection{Static Pressure}

Figure 7 presents the distribution of the static pressure in the longitudinal plane defined by $\mathrm{X}=0 \mathrm{~mm}$. According to these results, it has been noted that the static pressure is on its maximum in the inlet of the collector. Besides, it has been observed a depression above the obstacle. The static pressure decreases out of the test vein and a brutal drop of the pressure has been noted just behind the obstacle. The distribution of the static pressure shows that the depression zones characteristic of the minimum values are located in the second half of the wind tunnel through the diffuser. The comparison between the roof shape conforms that a brutal drop of the pressure has been observed just behind the inclined roof obstacle. This is due to the dead zone in this area which is more important than other shapes. 


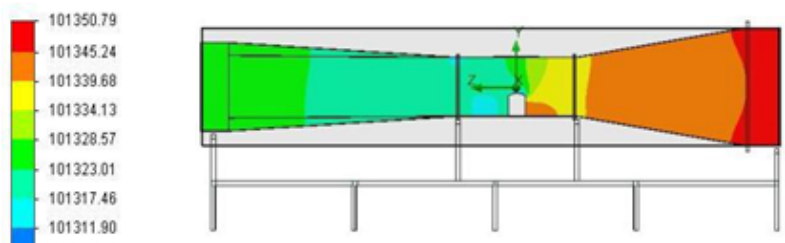

(a) Arched roof

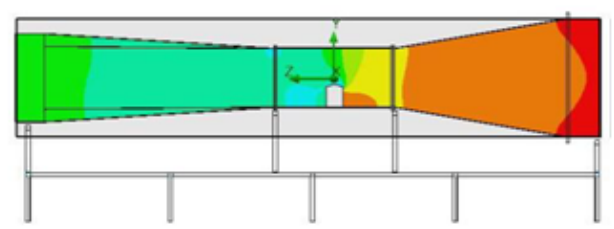

(c) Pitched roof

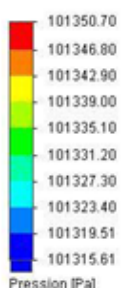

on $\mathbb{P}$ a]

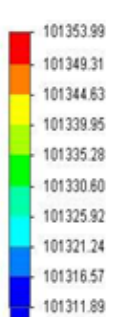

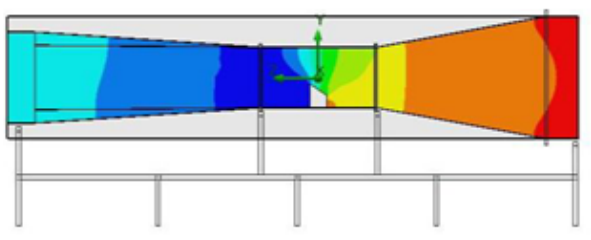

(b) Inclined roof

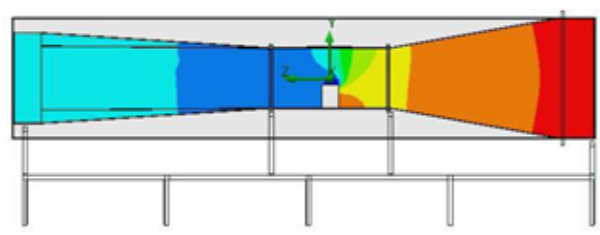

(d) Flat roof

Figure 7. Distribution of theStatic pressure

\subsection{Dynamic Pressure}

Figure 8 presents the distribution of the dynamic pressure in the longitudinal plane defined by $\mathrm{X}=0 \mathrm{~mm}$. According to these results, the dynamic pressure is found to be weak in the collector inlet and increases gradually through the collector as long as the tunnel section gets smaller. In the test section, the dynamic pressure keeps increasing in the upstream of the obstacle. Indeed, a
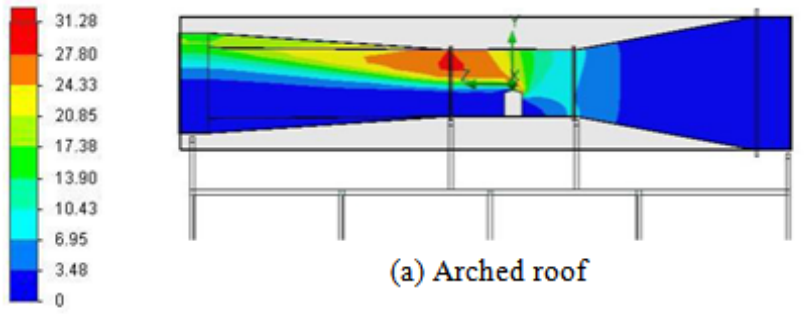

(a) Arched roof

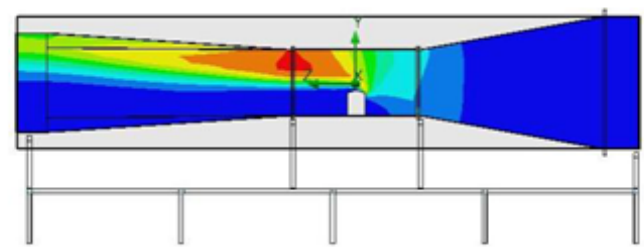

(c) Pitched roof compression zone is recorded in the region located behind the obstacle and is developed through the diffuser and in its upper side. However, the depression zone is located in the downside of the wind tunnel. In the considered gap, a wake characteristic of the maximum values appears in the obstacle downstream. The comparison between the roof shapes confirms that a brutal drop of the pressure appears just behind the inclined roof obstacle.
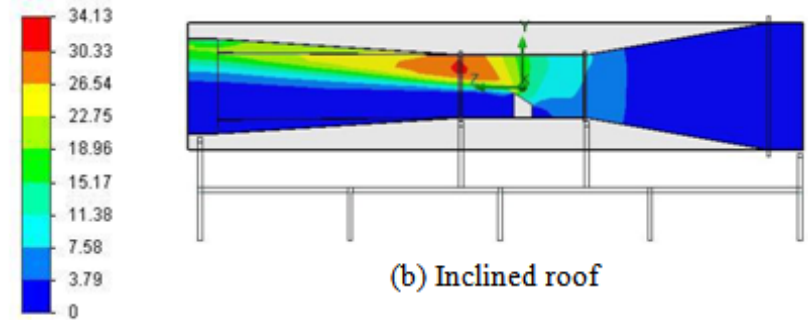

(b) Inclined roof

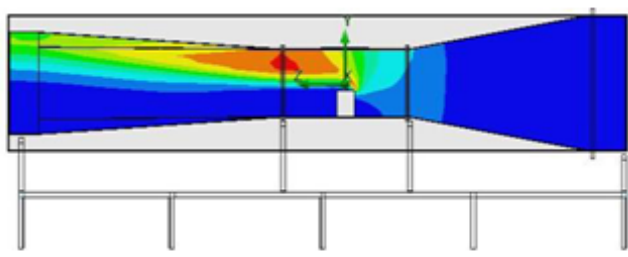

(d) Flat roof

Figure 8. Distribution of the Dynamic pressure

\subsection{Turbulent Kinetic Energy}

Figure 9 presents the distribution of the turbulent kinetic energy in the longitudinal plane defined by $\mathrm{X}=0$ $\mathrm{mm}$. From these results, it has been noted that the turbulent kinetic energy is weak in the first half of the wind tunnel in the obstacle upstream. A wake characteristic of the maximum value of the turbulent kinetic energy appears upstream of the obstacle. This wake starts in the obstacle corner until the outlet of the diffuser. The maximum value of the turbulent kinetic energy is found in the inclined roof obstacle model. For example, the turbulent kinetic energy is equal to $\mathrm{k}=2.4$ $\mathrm{m}^{2} . \mathrm{s}^{-2}$ with an inclined roof model while it's equal to $\mathrm{k}=1.94 \mathrm{~m}^{2} \cdot \mathrm{s}^{-2}$ with a flat roof model.

\subsection{Turbulent Dissipation Rate}

Figure 10 presents the distribution of the turbulent dissipation rate in the longitudinal plane defined by $\mathrm{X}=0$ $\mathrm{mm}$. From these results, it has been noted that the turbulent dissipation rate is very weak in the first half of the wind tunnel in the obstacle upstream. A wake characteristic of the maximum values of the turbulent dissipation rate appears upstream of the obstacle. This wake starts in the obstacle corner until the outlet of the diffuser and it's more important in the inclined roof model. In fact, the turbulent dissipation rate is equal to $\varepsilon=28.9$ W. $\mathrm{kg}^{-1}$ in the considered model and it's equal to $\varepsilon=22.7$ W. $\mathrm{kg}^{-1}$ in the pitched roof model. 


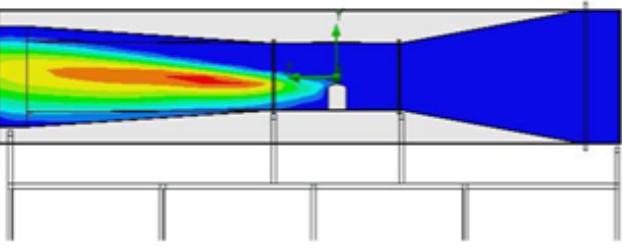

(a) Arched roof

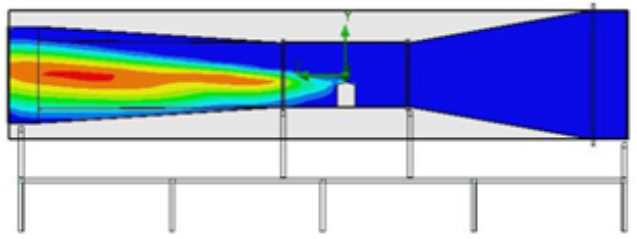

(c) Pitched roof
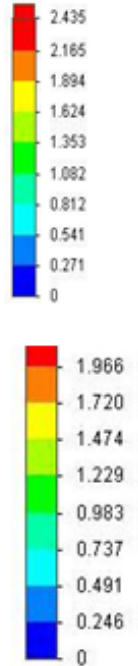

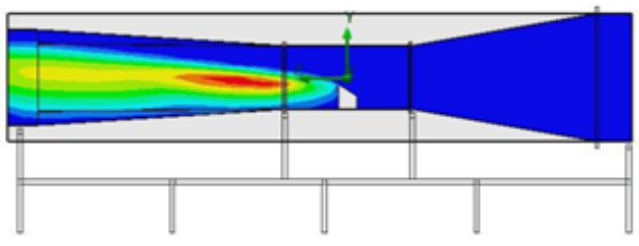

(b) Inclined roof

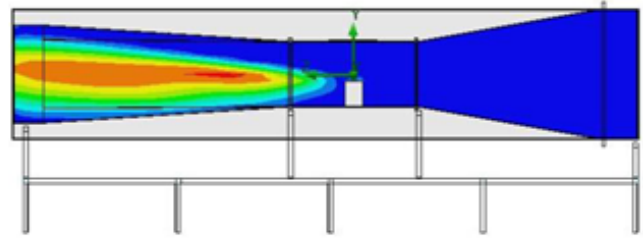

(d) Flat roof

Figure 9. Distribution of the turbulent kinetic energy

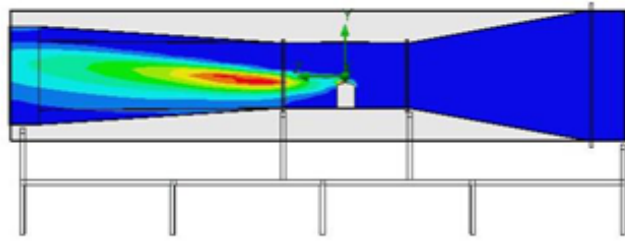

(a) Arched roof

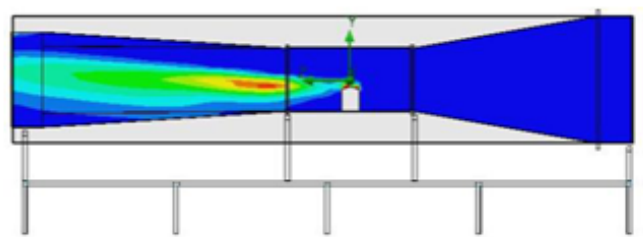

(c) Pitched roof
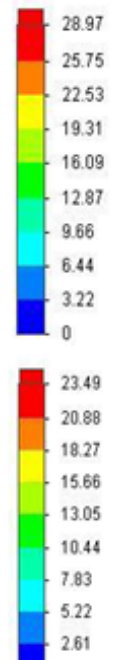

10

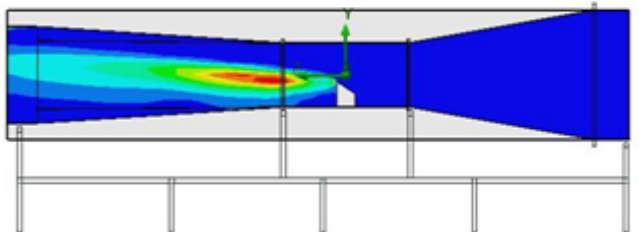

(b) Inclined roof

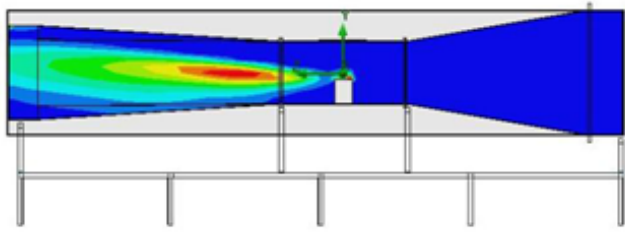

(d) Flat roof

Figure 10. Distribution of the turbulent dissipation rate

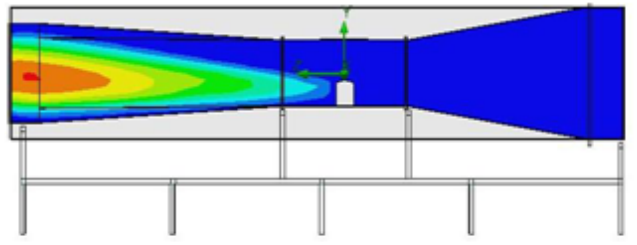

(a) Arched roof

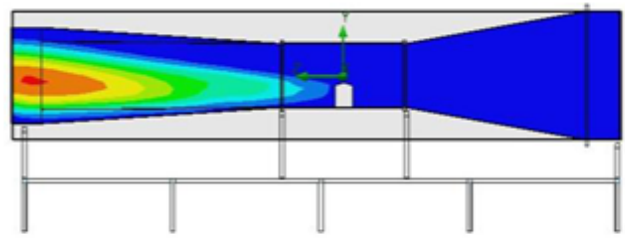

(c) Pitched roof
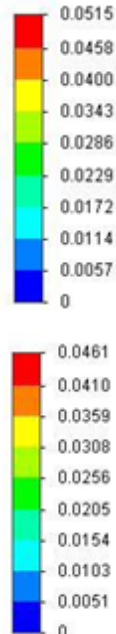

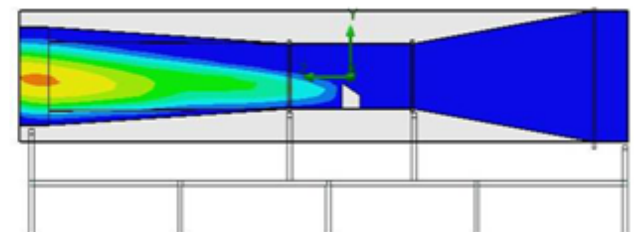

(b) Inclined roof

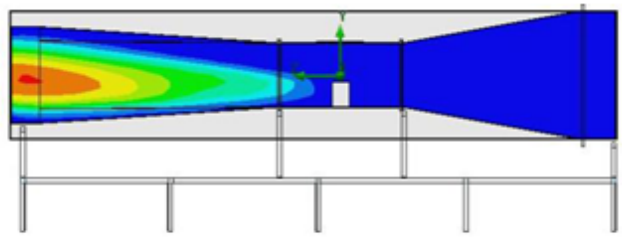

(d) Flat roof

Figure 11. Distribution of the turbulent viscosity

\subsection{Turbulent Viscosity}

Figure 11 presents the distribution of the turbulent viscosity in the longitudinal plane defined by $\mathrm{X}=0 \mathrm{~mm}$.
According to these results it has been noted that the viscosity is at its minimum in the collector region. After crossing the obstacle, the turbulent viscosity increases. The wake characteristic of the maximum values is located in the outlet of the diffuser. Indeed, it has been noted that 
the extension of the turbulent viscosity is more developed with the inclined roof. For example, with an arched roof obstacle model the turbulent viscosity is equal to $\mu_{\mathrm{t}}=0.044$ Pa.s. However, it's equal to $\mu_{\mathrm{t}}=0.045 \mathrm{~Pa}$.s with a pitched roof obstacle model.

\subsection{Vorticity}

Figure 12 presents the distribution of the vorticity in the longitudinal plane defined by $\mathrm{X}=0 \mathrm{~mm}$. According to these results, it's clear that the vorticity is weak in the collector region and starts increasing after crossing the obstacle. The wake characteristic of the maximum values of vorticity is reached after hitting the obstacle blade. The maximum value of the vorticity is found with the arched roof model. In this case, the maximum value is equal to $81.9 \mathrm{~s}^{-1}$ with the considered model. However, the maximum value of vorticity is equal to $66 \mathrm{~s}^{-1}$ with a flat roof obstacle model.

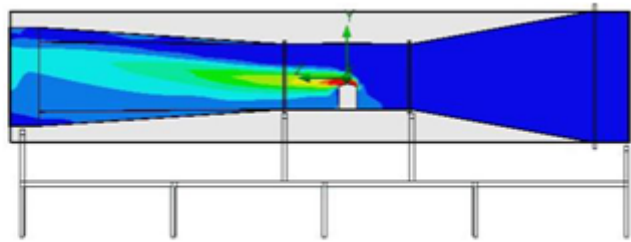

(a) Arched roof

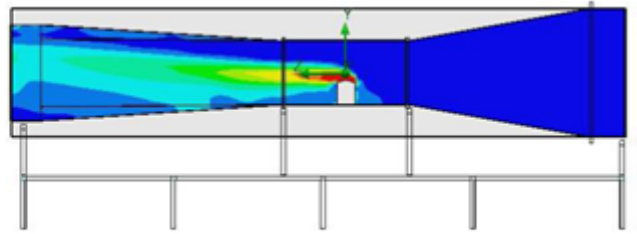

(c) Pitched roof

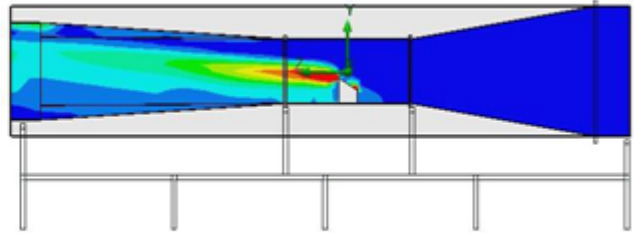

(b) Inclined roof

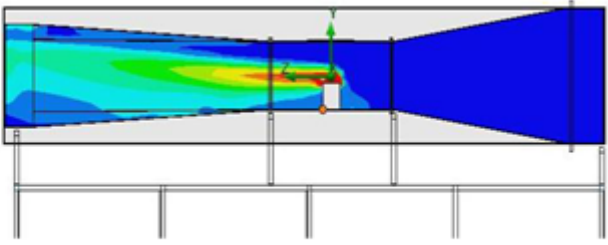

(d) Flat roof

Figure 12. Distribution of the vorticity

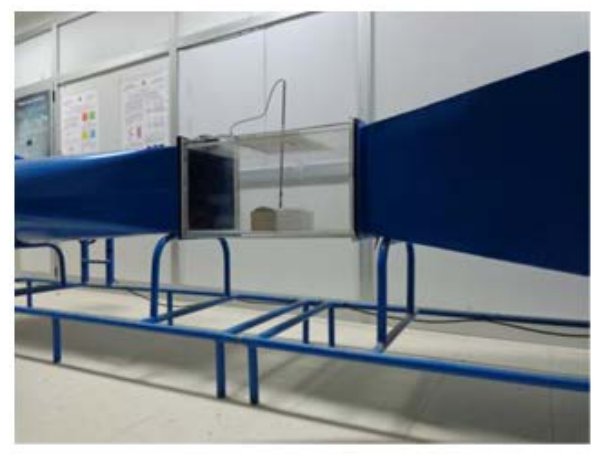

(a) Arched roof obstacle

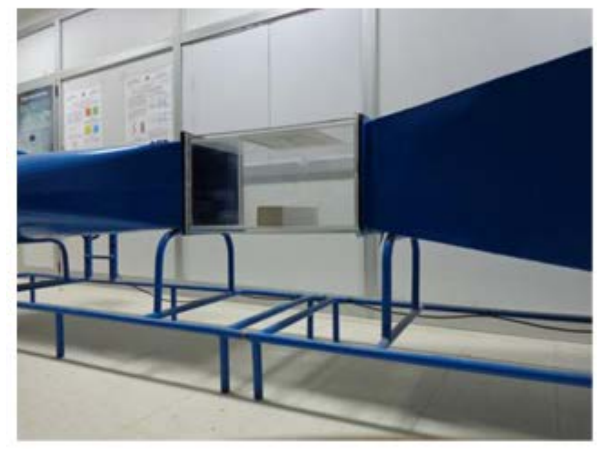

(c) Flat roof obstacle

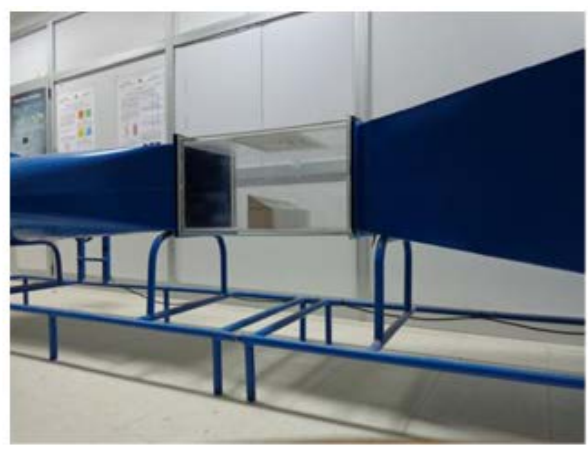

(b) Inclined roof obstacle

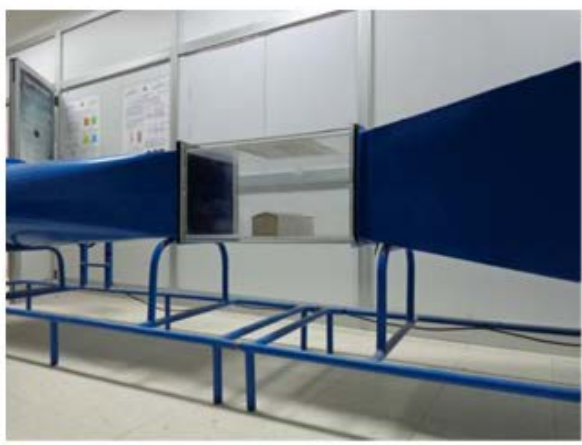

(d) Pitched roof obstacle

Figure 13. Prototypes

\section{Comparison with Experimental Results}

The wind tunnel results are used to validate the CFD model. The experiment is carried out in the atmospheric boundary layer wind tunnel at the National school of engineering of Sfax, Tunisia (Figure 13). The simulated data are inter polatedat the same grid points as in the wind tunnel experiment. As the configuration is replicated from the wind tunnel experiment, the CFD model tested using the mathematical model is validated against the data obtained from the wind tunnel experiments [22]. The 
results are shown in Figure 14. Values are taken along the direction defined by $\mathrm{X}=0 \mathrm{~mm}$ and $\mathrm{Z}=0 \mathrm{~mm}$. According to these results, it's clear that the velocity value is very weak near the obstacle. Outside, the velocity has the maximum value. The comparison between the numerical and experimental velocity values leads us to the conclusion that despite some unconformities, the values are comparable. The numerical model seems to be able to predict the aerodynamic characteristics of the air flow around the obstacle. The computational results tend to over-predict the velocity magnitude in comparison to

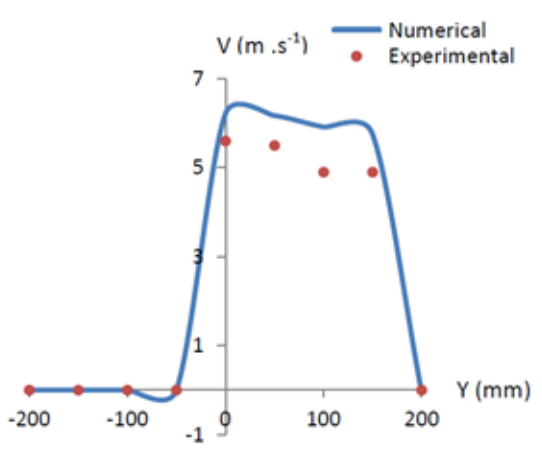

(a) Arched roof

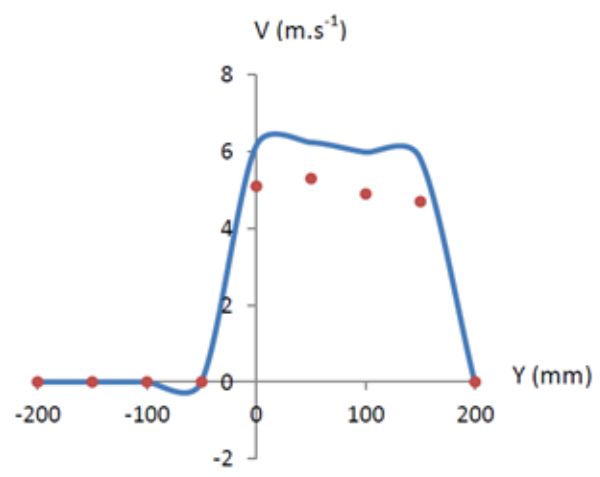

(c) Pitched roof experimental results. This fact is due to the using instrumentation. In fact, the velocity presents some fluctuations and cannot be measured exactly due to the anemometer insertion. In this situation, the used anemometry measures stable values in every fixed position. In the future, the Particle Image Velocimetry (PIV) system should be used. The CCD camera can be measure the velocity fields around the obstacle. This is due to the rapidity and the high technology of the PIV system. In this situation, the real value of the velocity should be measured in the chosen position.

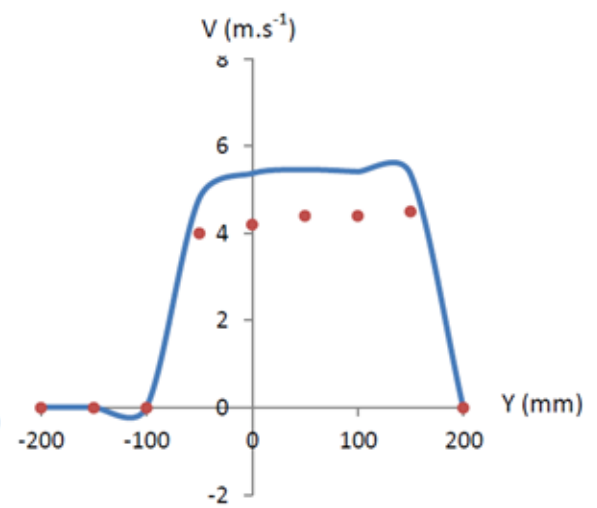

(b) Inclined roof

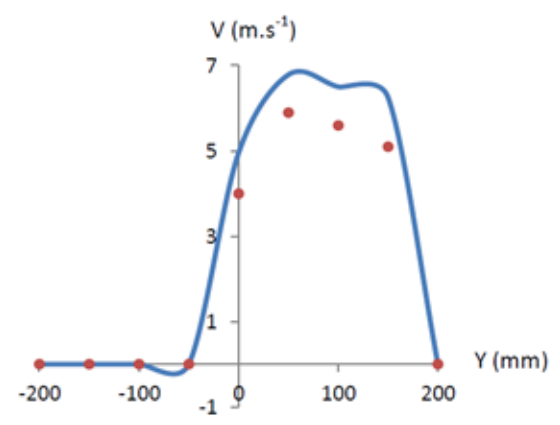

(d) Flat roof

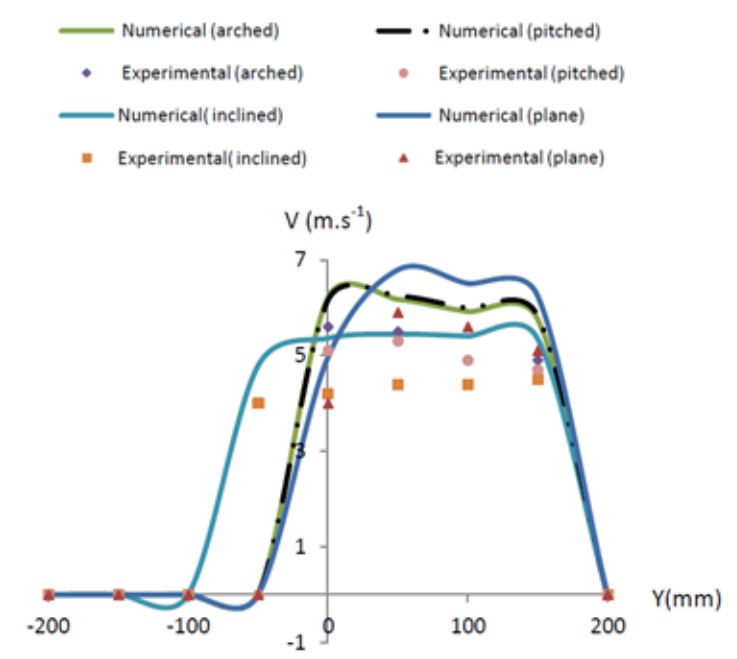

(e) Results superposition

Figure 14. Velocity in the direction defined by $\mathrm{z}=0 \mathrm{~mm}$ and $\mathrm{x}=0 \mathrm{~mm}$

\section{Conclusions}

This paper deals with the shape obstacle effect on the turbulent flow. Based on the results obtained so far, we have noted that the maximum value of the magnitude velocity was found in the inclined roof obstacle model. Indeed, a brutal drop of the pressure has been observed just behind the inclined roof obstacle. This is due to the dead zone in this area which is more important than other 
shapes. The maximum value of the turbulent kinetic energy and the turbulent dissipation rate is found with the inclined roof obstacle mode. However the maximum value of the vorticity is found with the arched roof model.

These phenomena and recommendations have to be considered during the building design location and installation.

\section{Nomenclature}

\section{l length, $\mathrm{m}$}

$\mathrm{p}$ straight edge, $\mathrm{m}$

$\mathrm{P}$ pressure, $\mathrm{Pa}$

Re Reynolds number

$\mathrm{s}$ bucket length, $\mathrm{m}$

$\mathrm{t}$ time, $\mathrm{s}$

$\mathrm{V}$ velocity magnitude, $\mathrm{m} . \mathrm{s}^{-1}$

$\mathrm{x} \quad$ Cartesian coordinate, $\mathrm{m}$

y Cartesian coordinate, $\mathrm{m}$

$\mathrm{z} \quad$ Cartesian coordinate, $\mathrm{m}$

$\varepsilon \quad$ dissipation rate of the turbulent kinetic energy, W.kg-1

$\mu \quad$ dynamic viscosity, Pa.s

$\mu_{\mathrm{t}} \quad$ turbulent viscosity, Pa.s

$\rho$ density, kg. $\mathrm{m}^{-3}$

$\sigma_{\mathrm{k}} \quad$ constant of the k- $\varepsilon$ turbulence model

$\sigma_{\varepsilon} \quad$ constant of the k- $\varepsilon$ turbulence model

\section{References}

[1] Barlow, J.F., Coceal, O., "A review of urban roughness sublayer turbulence," Met. Office Technical Report, 527. 2009.

[2] Belcher, S.E., "Mixing and transport in urban areas," Phil. Trans. $R$ Soc., A 363. 2005.

[3] Ntinas, G.K., Zhang, G., Fragos, V.P., Bochtis, D.D., NikitaMartzopoulou, C., "Airflow patterns around obstacles with arched and pitched roofs: Wind tunnel measurements and direct simulation," European Journal of Mechanics B/Fluids, 43. 216229. 2014.

[4] Luo,W., Dong,Z., Qian, G., Lu, J., "Wind tunnel simulation of the three-dimensional airflow patterns behind cuboid obstacles at different angles of wind incidence, and their significance for the formation of sand shadows," Geomorphology, 139-140. 258-270. 2012.

[5] Ahmed, K., Khare, M., Chaudhry, K.K., "Wind tunnel simulation studies on dispersion at urban street canyons and intersections- a review,” J. Wind Eng. Ind. Aerodyn., 93. 697-717. 2005.

[6] Jiang, Y., Alexander, D., Jenkins, H., Arthur, R., Chen, Q., "Natural ventilation in buildings: measurement in a wind tunnel and numerical simulation with large-eddy simulation, "J. Wind Eng. IndAerodyn, 91.331-353. 2003.
[7] K. Nozawa, T. Tamura, "Large eddy simulation of the flow around a low-rise building immersed in aa rough-wall turbulent boundary layer,” J. Wind Eng. Ind. Aerodyn, 90. 1151-1162. 2002.

[8] Becker, S., Lienhart, H., Durst, F., "Flow around threedimensional obstacles in boundary layers," J. Wind Eng. Ind. Aerodyn., 90. 265-279, 2002.

[9] Ikhwan, M., Ruck,B., "Flow and pressure field characteristics around pyramidal buildings," J. Wind Eng. Ind. Aerodyn, 94. 745765, 2006.

[10] Kenjeres, S.,Cate, S., Voesenek, C.J., "Vortical structures and turbulent bursts behind magnetic obstacles in transitional flow regimesn," International journal of heat and fluid flow, 32. 510528. 2011.

[11] Santos, J.M., Reis, N.C., Goulart, E.V., Mavroidis, I., "Numerical simulation of flow and dispersion around an isolated cubical building: The effect of the atmospheric stratification", Atmospheric Environment, 43. 5484-5492. 2009.

[12] Franke, J., Sturm, M., Kalmbach, C., "Validation of Open FOAM 1.6. $x$ with the German VDI guideline for obstacle resolving microscale models," J. Wind Eng. Ind. Aerodyn., 104-106. 350-359, 2012.

[13] SadaK., Sato, A., "Numerical calculation of flow and stack-gas concentration fluctuation around a cubical building," Atmospheric Environment, 36. 5527-5534. 2002.

[14] Y. Tominaga, T. Stathopoulos, "CFD Simultation of near-field pollutant dispersion in the urban environment: A review of current modeling techniques," Atmospheric Environment, 79. 716-730. 2013.

[15] De Paepe, M., Peters, J.G., Cornelis, W.M., Gabriels, D., Merci, B., Demeyer, P., "Airflow measurements in and around scalemodel cattle barns in a wind tunnel: Effect of wind incidence angle,” Biosystems Engineering, 115. 211-219. 2013.

[16] Lim, H.C., Thomas, T.G., Castro, L.P., Flow around a cube in a turbulent boundary: "Les and experiment," J. Wind Eng. Ind. Aerodyn., 97. 96-109, 2009.

[17] De Melo, A.M.V., Santos, J.M., Mavroidis, I., "Neyval Costa ReisjuniorModelling of odour dispersion around a pig farm building complex using AERMOD and CALPUFF. Comparison with Wind tunnel results," Building and Environment, 56.8-20, 2012.

[18] Driss Z., Mlayeh, O., Driss, D., Maaloul, M, Abid, M.S., "Numerical simulation and experimental validation of the turbulent flow around a small incurved Savoniuswind rotor," Energy, 74. 506-517, 2014.

[19] Ammar, M., Chtourou, W., Driss, Z., Abid, M.S. "Numerical investigation of turbulent flow generated in baffled stirred vessels equipped with three different turbines in one and two-stage system,” Energy, 36.5081-5093. 2011.

[20] Driss, Z., Ammar, M., Chtourou, W., Abid, M.S. "CFD Modelling of Stirred Tanks," Engineering Applications of Computational Fluid Dynamics, 5. 145-258,2011.

[21] Driss, Z., Abid, M.S. "Use of the Navier-Stokes Equations to Study of the Flow Generated by Turbines Impellers," NavierStokes Equations: Properties, Description and Applications, 3.51138. 2012.

[22] Driss, S., Driss, Z., KallelKammoun, I., "Study of the Reynolds Number Effect on the Aerodynamic Structure around an Obstacle with Inclined Roof,” Sustainable Energy, 2 (4). 126-133, 2014.

[23] SolidWorks, "Enhanced turbulence modeling in SolidWorks flow simulation," DassaultSystèmesSolidWorks Corporation Waltham, USA, MKTURBMODWPENG0313, 2013. 